\title{
Retour sur « L'Analyse praxéologique »
}

\section{Claudine de France}

\section{OpenEdition}

Journals

Édition électronique

URL : https://journals.openedition.org/tc/5151

DOI : $10.4000 /$ tc. 5151

ISSN : 1952-420X

\section{Éditeur}

Éditions de l'EHESS

\section{Édition imprimée}

Date de publication : 30 juin 2010

Pagination : 220-222

ISSN : 0248-6016

\section{Référence électronique}

Claudine de France, «Retour sur « L'Analyse praxéologique »», Techniques \& Culture [En ligne], 54-55 |

2010, mis en ligne le 30 janvier 2013, consulté le 29 septembre 2022. URL : http://

journals.openedition.org/tc/5151 ; DOI : https://doi.org/10.4000/tc.5151 


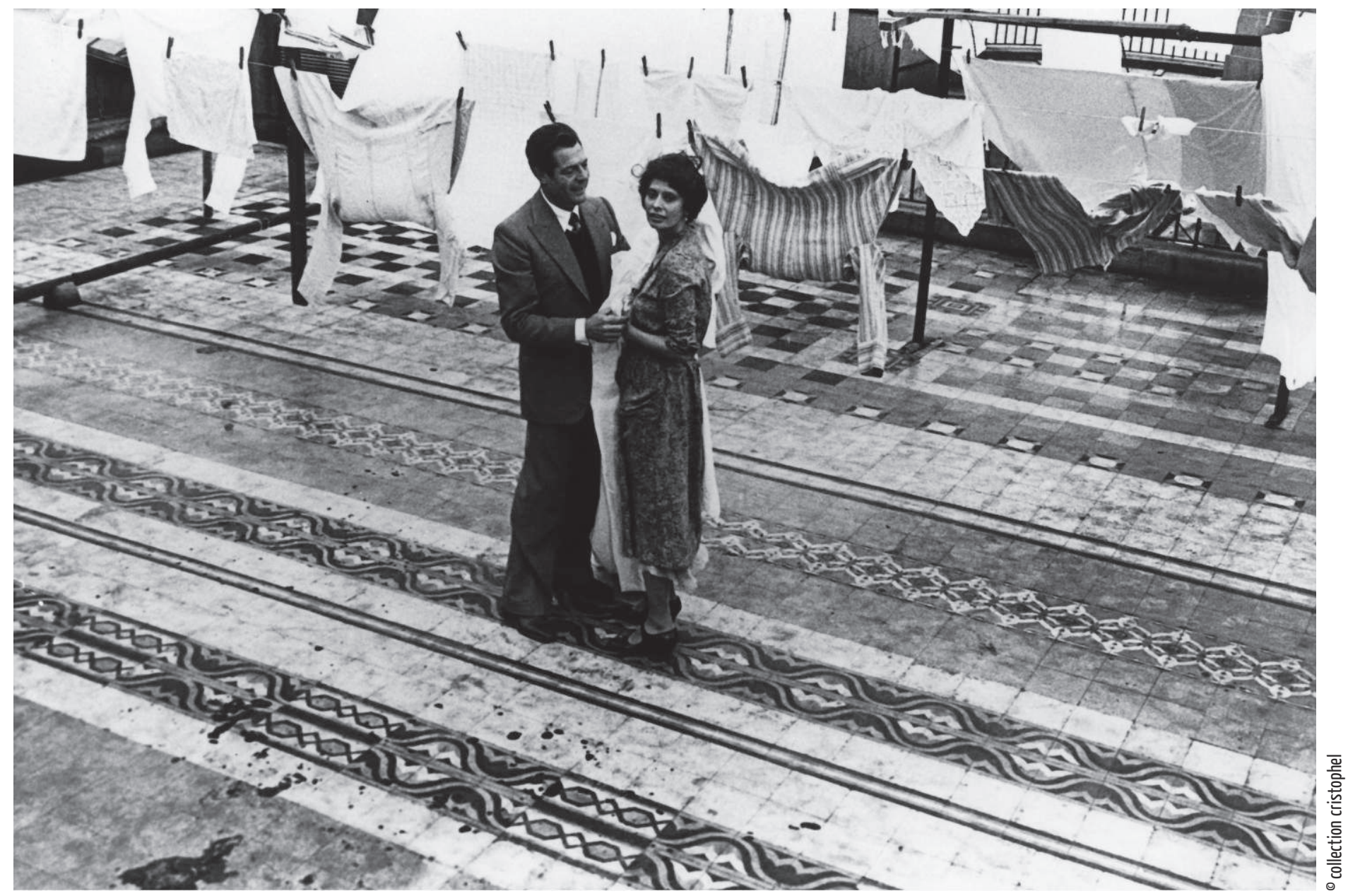




\section{« L’Analyse praxéologique»}

Lorsque fut écrit le présent article, il y a bientôt 30 ans, l'usage du cinéma était suffisamment répandu parmi les anthropologues, à la suite de Jean Rouch en France, pour susciter des interrogations de plus en plus poussées sur ses fondements, ses outils, ses méthodes, son champ d'application.

D’autre part, le « comportement technique » tel que l'avait défini André Leroi-Gourhan - faisant ainsi de la technologie une science humaine - me paraissait constituer une matière privilégiée de l'appréhension cinématographique.

À partir de là, l'ensemble des actions sensibles, apparentes, impulsées par le geste en son milieu ne pouvait-il être revisité par le regard de l'anthropologue-cinéaste armé de sa caméra, effectuant ici et là, dans l'espace et dans le temps, des coupures, des enchâ̂nements que n'opéraient pas toujours le langage ou l'observation directe? Un éclairage nouveau pouvait être ainsi apporté sur la manière dont s'agençaient, dans l'espace et dans le temps, les processus techniques que l'on filmait.

C'est donc sous l'angle de l'espace et du temps, considérés comme une forme qualitative de l'action captée par l'observation filmique, qu'a été conçue la méthode d'analyse praxéologique ici exposée.

Ma réflexion s'est principalement appuyée sur des films que j'ai réalisés pour la plupart en tournage discontinu et en son postsynchronisé, au début des années 1970. Or les rigueurs de l'instrumentation audiovisuelle, produisant des images muettes d'une durée limitée, avaient pour avantage d'attirer l'attention sur les gestes des personnes filmées que les fréquentes coupures de l'enregistrement imposées par la caméra sacrifiaient constamment. Cela m'a conduite à rechercher des compensations filmiques à ces absences et, plus encore, à m'interroger sur un «non-montré » de l'image qui pouvait se révéler indispensable à la compréhension de l'action. 
Qu'attendre aujourd'hui d'une analyse praxéologique conçue dans ces conditions, alors que les techniques d'enregistrement vidéographiques et vidéonumériques ont décuplé les possibilités d'appréhension du continuum d'action? De nos jours, la parole a été donnée en abondance aux personnes filmées, de sorte qu'entretiens et dialogues envahissent et parfois dominent l'espace et le temps du présenté. Lors de l'enquête filmique, l'anthropologue-cinéaste n'appréhende plus le geste, l'action matérielle, uniquement pour eux-mêmes, mais il tient compte de la représentation que les personnes filmées en ont, du sens qu'elles leur accordent. Les coupures qu'il introduit dans l'image sont alors plus dépendantes de la parole que des gestes des êtres filmés. En outre, la crainte d'une chosification de ces mêmes êtres filmés éloigne le chercheur d'une exploitation classifiante de leurs actions.

Cependant l'introduction progressive du multimédia dans la présentation des données filmées incite à porter un regard encore plus attentif sur leur contenu et sur la manière dont elles sont enregistrées et mises en scène. Où couper? quand couper? sont des questions plus que jamais décisives pour l'anthropologue-cinéaste. Ainsi peut être envisagée une forme d'analyse qui, attachée jusqu'ici à l'action " filmée », se déporterait vers l'action « filmante » du cinéaste. Cadrages, angles de vue, durée des plans, seraient de ce fait considérés en fonction de leurs relations de composition, d'ordre et d'articulation dans l'espace et le temps. Pourrait être alors entreprise une étude de la coordination entre les modes de l'action filmée et de l'action filmante au sein du film.

Par ailleurs, lors du brassage en tous sens des flux d'images qu'offre la présentation multimédia, il devient possible d'opérer des découpages à l'infini entre de grands ensembles d'actions filmées facilitant mises en relation et comparaisons, suggestives ou démonstratives. Encore faut-il s'assurer que ces découpages n'obéissent pas au seul souci d'inventaire, fondé sur les compartimentages propres au langage. Ici peut intervenir une analyse praxéologique, certes enrichie et quelque peu transformée, mais toujours soucieuse de démêler ce qui relève des relations spatio-temporelles telles que nous les avons envisagées entre les flux d'actions qu'offrent les images.

Quant aux contenus brassés par ces nouvelles formes de présentation, ils mettent à nu la trame sensible d'un « faire » toujours présent, manifestation première du social. Avec ses contraintes et ses options, cette trame sensible est le tremplin indispensable à l'étude de la plupart des processus d'ordre non sensible qui prennent appui sur elle. Dans ces conditions, il importe de donner sa juste place, en persistant à le filmer et l'analyser, au continuum technico-rituel qui ne cesse d'être là sous nos yeux mais que l'on ne sait pas toujours voir, tout comme La Lettre volée de la nouvelle d'Edgar Poe. 